\title{
MENTES EN CUERPOS DE AFECTO: NOTA CRÍTICA SOBRE ECOLOG Y OF THE BRAIN, DE THOMAS FUCHS
}

Thomas Fuchs, Ecology of the Brain, Oxford University Press, Oxford, 2018, 336 pp.

LAURA MOJICA Okinawa Institute of Science and Technology

Universidad Autónoma Metropolitana Unidad Cuajimalpa laura.mojica460@gmail.com

\section{Introducción}

La idea de que la mente es equiparable al cerebro es extremadamente popular hoy en día. Se asume como verdad indiscutible que los fenómenos como la percepción, la conciencia y la resolución de problemas son esencialmente procesos cerebrales. Esta idea se materializa, por ejemplo, en la psiquiatría, que equipara desórdenes afectivos con desbalances en el cerebro, o en posiciones de filosofía de la mente y ciencias cognitivas que asumen, por ejemplo, que la percepción es esencialmente una representación cerebral del mundo exterior o que la información es procesada y representada por módulos cerebrales dedicados a diferentes tareas como el procesamiento del lenguaje o las respuestas emocionales. Ecology of the Brain de Fuchs es una propuesta refrescante dentro de este panorama que, sin desconocer la importancia del cerebro, vuelve a otorgar un sitio protagónico dentro de la cognición al sujeto corporizado, situado en su ambiente e inequiparable con su cerebro.

Ecology of the Brain ofrece una explicación empíricamente sustentada del papel del cerebro en la cognición desde una perspectiva enactiva, corporizada, extendida y embebida (Fuchs 2018, p. 108) - perspectivas también conocidas como 4e cognition: embodied, embedded, enacted, extended, en las que se entiende la cognición como un proceso de interacción entre un organismo y su ambiente- Para Fuchs, la cognición es 1) corporizada en la medida en que su realización en un cuerpo vivo es constitutiva de su consecución; 2) es embebida porque "los sistemas cognitivos explotan sus circunstancias 
específicas para incrementar sus capacidades" (p. 108); ${ }^{1} 3$ ) es extendida más allá de los límites del cuerpo en cuanto que los sistemas cognitivos están conectados inherentemente con su ambiente por medio de bucles de retroalimentación, y 4) es enactiva porque surge sólo en la interacción activa entre un agente y su ambiente (p. 108). Fuchs argumenta que el agente cognitivo corporizado y su perspectiva subjetiva de primera y segunda personas son irreducibles a las funciones cerebrales incluso a la hora de construir una explicación científica de la cognición. Desde esta perspectiva y sin desconocer la importancia del cerebro, propone que éste tiene tres funciones fundamentales: (i) es un órgano de mediación y transformación en la interacción sensoriomotora (p. 131); (ii) es un órgano de regulación e integración de los procesos vegetativos, y sensoriomotores (p. xviii), y (iii) es un órgano de interrelaciones, capaz de aprendizajes sinápticos y de guardar rastros en el nivel neuronal de las interacciones con otros (p. 181). Estas funciones, argumenta Fuchs, dependen de que exista un bucle de retroalimentación constante entre cerebro, cuerpo y medio ambiente, y no una relación de causalidad lineal. En otras palabras, el cerebro cumple su papel porque en él constantemente resuenan tanto las dinámicas internas del resto del organismo como las dinámicas externas del ambiente.

El papel del cerebro en la cognición que Fuchs propone es un aporte novedoso y necesario no sólo para las ciencias cognitivas radicalmente corporizadas, sino también para el debate más amplio de las disciplinas que se dedican a explicar la mente, como la psicología, la psiquiatría y la filosofía de la mente. Ecology of the Brain presenta argumentos sólidos contra la concepción de la explicación como reducción en la filosofía de la mente y las ciencias cognitivas, es decir, contra la idea de que explicar la mente es reducirla a procesos computacionales o neuronales. De manera que su propuesta es también una contribución a la pregunta abierta acerca de cuál es el mejor marco explicativo para la mente.

En su libro, Fuchs logra al menos otras tres aportaciones importantes. En primer lugar, ofrece un argumento negativo contra los intentos de explicar la mente desde una perspectiva puramente de tercera persona. Por un lado, argumenta que el significado es una relación entre significante, significado y para quién significa, que se derrumba si se reduce a la relación impersonal entre significante, por ejemplo, input de dolor, y significado, por ejemplo, output comportamental de dolor. Dicho de otro modo, ni el dolor ni ninguna

${ }^{1}$ La traducción de ésta y todas las demás citas del texto son de la autora. 
percepción existen si nadie vive la experiencia, y, por ende, no hay mente si no hay experiencia subjetiva de mundo significativo. Fuchs sostiene, además, que la subjetividad no se trata de una colección de estados mentales, digamos, la sensación de azul o de calor, sino que es esencialmente una "orientación hacia lo que no es uno mismo: [la subjetividad] está abierta al mundo, relacionada con objetos y dirigida hacia contenidos y significados" (p. 36), de manera que es imposible que haya intencionalidad sin experiencia significativa. $\mathrm{Y}$ puesto que es imposible que haya estados mentales completamente funcionales sin experiencia subjetiva, el problema difícil de la conciencia no surge bajo esta concepción de mente. Este argumento logra recopilar, además, de forma sucinta y articulada críticas bien conocidas a la idea de reducir la mente al cerebro o a sus operaciones funcionales (pp. 3739) - por ejemplo, las de Searle, Zahavi, Strawson, Ryle, Bennett y Hacker-, y las lleva a una de sus consecuencias más radicales: estos intentos explicativos buscan describir la mente desde la tercera persona y con ello desconocen el papel fundamental de la subjetividad y la relación de primera persona que los agentes cognitivos establecemos con el mundo. En segundo lugar, su propuesta hace justicia en sus cimientos al papel esencial de la afectividad y de la intersubjetividad en la constitución de la cognición. En tercer lugar, transforma el tradicional e irresoluble problema mente-cuerpo o mente-cerebro en una característica de los cuerpos vivos que puede ser abordada desde la fenomenología: la aspectividad dual (dual aspectivity). Este concepto captura, por un lado, que el cuerpo es el centro de la experiencia y la subjetividad del ser vivo: en esa medida, es un cuerpo vivido; por otro lado, ese mismo cuerpo se puede ver como un objeto físico, vivo, compuesto por procesos y con características susceptibles de ser estudiadas: en esa medida, es un cuerpo vivo.

En esta reseña me centraré en la segunda de estas contribuciones; discutiré la concepción de afectividad de Fuchs y el papel que le atribuye en la cognición. Empezaré ofreciendo un resumen del argumento central de Ecology of the Brain que sentará las bases para discutir, en las últimas dos secciones, las propuestas de Fuchs sobre afecto y emociones con mayor profundidad.

\section{Cerebro, organismo y ambiente: causalidad circular}

La concepción que defiende Fuchs de sujeto cognitivo como organismo vivo e interactivamente situado en su ambiente depende de que las relaciones causales a las que se somete no se entiendan de manera lineal. Fuchs propone dos tipos de causalidad que capturan 
las relaciones bajo las que opera el organismo, incluido el cerebro: causalidad circular vertical y causalidad circular horizontal.

En primer lugar, la causalidad circular vertical ocurre dentro del organismo y entre los diferentes niveles de elementos que lo componen: átomos, células, órganos y el propio organismo como un todo. Esta causalidad no ocurre entre dos entidades distintas, sino entre un todo y sus partes, y en ambas direcciones. Primero, cada nivel, es decir, cada célula, órgano, organismo completo, etc., determina los procesos del nivel inferior porque abre y cierra posibilidades a los elementos de este nivel en función del papel que cumplen dentro del nivel superior al que pertenecen. Esta relación causal es de arriba abajo o top-down. Por otro lado, cada nivel existe y es capaz de llevar a cabo procesos porque los elementos y subprocesos que lo componen se organizan de manera que en conjunto constituyen tal nivel superior. Los procesos mentales, en cuanto actos de un organismo vivo, y la neurofisiología del agente se relacionan precisamente de esta forma. Fuchs ejemplifica este punto esbozando una explicación de un acto de habla: la causa más próxima del movimiento de los músculos de la lengua y la laringe es la liberación de acetilcolina, pero éstos se organizan en patrones de movimiento porque el agente está diciendo ciertas palabras y está orientado intencionalmente hacia su contenido (para una explicación operacional y detallada de cómo se organizan las estructuras coordinativas en función de la intencionalidad en la producción del habla, véase Kelso et al. 1984). En este caso, la intencionalidad del agente actúa como causa de orden superior que selecciona cuáles de los posibles movimientos de los músculos serán ejecutados y forma una dinámica del conjunto total de los músculos, esto es, causalidad top-down. Asimismo, el acto intencional del agente selecciona y da forma a los patrones de activación neuronal, que en conjunto con las demás dinámicas fisiológicas conforman el acto mismo de habla; esto es causalidad bottom-up. Puesto que la relación entre el todo y las partes es bidireccional, Fuchs la cataloga como causalidad circular vertical.

De acuerdo con Fuchs, las funciones características del cerebro se pueden explicar en términos de causalidad circular vertical y horizontal. En primer lugar, "el cerebro funciona como un transformador de la causalidad circular vertical" (p. 98) que integra y regula los procesos de distintos niveles, el vegetativo, el endocrino y el sensoriomotor. Esa función permite transformar "estados globales o de alto nivel [por ej., la intencionalidad] y microestados de bajo nivel [por ej., los neuroquímicos] y, en cada caso, los vuelve efectivos en el otro nivel jerárquico" (p. 98). En otras palabras, el cerebro, al 
integrar y regular procesos de distintos niveles, flexibiliza y posibilita la influencia causal de niveles superiores a inferiores, y viceversa. En esta relación circular causal vertical surge la subjetividad básica que se caracteriza por el sentimiento primario de estar vivo. En la siguiente sección veremos que este sentimiento primario además de ser la fuente de la vida afectiva, es fundamental para la constitución de la cognición. En segundo lugar, la causalidad circular horizontal ocurre en la interacción organismo-ambiente. Esta relación captura el bucle de influencia causal entre cambios en el ambiente y cambios en el organismo que se repite y acomoda hasta que llega a un punto de equilibrio. Operacionalmente, esto significa que la interacción agente-ambiente, por ejemplo, percibir un animal a lo lejos o montar en bicicleta, es un sistema dinámico que tiende a ciertos patrones de oscilación, es decir, atractores, que constituyen sus puntos de equilibrio (p. 149). Los cambios que se producen en este tipo de interacciones no se pueden explicar como una relación lineal, porque son cambios cualitativos que ocurren en función de la organización del sistema vivo.

El cerebro, propone Fuchs, desempeña dos papeles en esta interacción: (i) la media y la transforma porque permite al organismo retrasar y modificar su respuesta motora ante los estímulos sensoriales, y (ii) es capaz de "guardar" patrones de activación sináptica de tales interacciones de manera que se activen más fácil y rápidamente respuestas complejas de coordinación cerebro-cuerpo-ambiente en ocasiones futuras, es decir, en términos operacionales, permite la creación de atractores en la actividad sináptica. Esta capacidad es crucial para el desarrollo de la interacción con otros y para el desarrollo emocional en la medida en que permite al sujeto reconocer aspectos con carga de valor en el ambiente e interactuar adecuadamente con ellos (pp. 141, 181).

Según Fuchs, la descripción de estas interacciones causales sólo captura uno de los dos aspectos esenciales del sujeto cognitivo, el aspecto de su corporalidad como organismo viviente que puede ser descrito desde la perspectiva de tercera persona. Este aspecto existe únicamente como una de las caras de la moneda del sujeto, la otra es su corporalidad como centro de experiencias del mundo y de interacciones con el otro. En fenomenología, a este último aspecto se le conoce como cuerpo vivido y, para Fuchs, capta la unidad esencial de la que se puede decir, con sentido, que es sujeto de experiencias, que conoce, que cree y que interactúa con el mundo. La corporalidad vivida es, para Fuchs, el centro primario e irreducible de la subjetividad y la conciencia que, por nuestra naturaleza de organismos vivientes y 
vividos, es inescapable: ni siquiera los intentos por describir de manera naturalista la mente pueden escapar a la perspectiva de primera persona desde la que tiene sentido vivir fenómenos de la conciencia, ni a la segunda persona desde la que adscribimos una vida mental a otros inmediatamente en la interacción y sin la mediación de cálculos impersonales.

Ninguna descripción puramente desde la tercera persona podría dar cuenta de la mente y de las relaciones significativas que establecemos con el mundo y con otros por dos razones. En primer lugar, no hay fundamento bajo esta descripción desapegada para separar los fenómenos mentales de los demás fenómenos de la naturaleza. En segundo, la naturaleza propia de la mente implica que su constitución y su desarrollo dependen esencialmente de que exista un sujeto corporizado capaz de vivenciar, desde la perspectiva de primera persona, sus interacciones con el mundo, y de interactuar con otros como seres vivos, no como meros objetos equiparables a las máquinas o las piedras; en otros términos, tener mente implica ser un sujeto capaz de interactuar desde la segunda persona. De esta manera, Fuchs argumenta que los sujetos cognitivos tienen inherentemente una aspectividad dual, como cuerpos vividos y como cuerpos vivientes, y que su naturaleza como sujetos vividos es irreducible a su naturaleza como seres vivientes; es decir, la experiencia es irreducible a la descripción puramente desapegada desde la perspectiva de tercera persona.

Concebir al sujeto cognitivo de esta forma corporizada e interaccional tiene consecuencias para la forma en que se explican y tratan los desórdenes mentales que Fuchs explora en el capítulo final de su libro. En general, un desorden mental es una perturbación tanto en la causalidad circular vertical que constituye al organismo, es decir, en los procesos neurológicos y fisiológicos de nivel inferior y su relación con la experiencia subjetiva del agente, como en la horizontal que constituye al organismo en interacción con su ambiente y con los otros. Mientras otras enfermedades se viven como un distanciamiento de partes del cuerpo que se presentan como obstáculos para el sujeto, en las enfermedades mentales la experiencia misma de ser un sujeto está alterada, de manera que el desorden no se puede localizar en ninguna parte del cuerpo. Fuchs ejemplifica este punto con la depresión: se detona por una situación vivida y percibida como una amenaza que no se puede superar, y se caracteriza por ser un bucle causal entre estrés fisiológico permanente (sobreactivación del sistema simpático, de la producción de cortisol y la desregularización de la transmisión de serotonina) y la experiencia vivida corporalmente de 
agotamiento y opresión. Puesto que la depresión, como cualquier desorden mental, es un trastorno de los procesos neurofisiológicos e interaccionales, no se puede predecir atendiendo a un solo elemento (por ej., el tiempo de recaptación de serotonina, los genes, el desarrollo), sino que se deben contemplar los procesos de causalidad circular vertical y horizontal. En particular, Fuchs sostiene que si bien los polimorfismos genéticos aumentan la sensibilidad al estrés (de Kloet et al. 2005, citado en Fuchs 2018, p. 264), éstos no se convierten en un factor etiológico hasta que son parte de la causalidad circular vertical formada en el cuerpo por las experiencias vividas del estrés y de la causalidad circular horizontal en la que el agente es incapaz de formar relaciones favorables con otros. Este tipo de relaciones, sostiene Fuchs apoyado en el trabajo de Kendler y colegas (Fuchs 2018, p. 264), se ven favorecidas por interacciones tempranas en las que no hay un entrenamiento adecuado en competencias sociales y fomentan autoevaluaciones negativas y visiones del mundo que favorecen la depresión: profecías catastróficas autocumplidas, sentimientos de culpa, etc. En suma, a la hora de identificar las vulnerabilidades de un sujeto a la depresión es necesario reconocer la interacción compleja entre procesos causales circulares de la que forman parte crucial los patrones de evaluación de estresores y la experiencia subjetiva del agente.

\section{Afecto, emociones y subjetividad}

En el resto de esta reseña me concentraré en el papel que Fuchs atribuye a la afectividad en la cognición, y en las posibilidades de investigación que esto abre, no sólo porque en el marco de su propuesta aquélla cumple un papel fundamental, sino porque es uno de los aspectos más novedosos y fecundos de sus planteamientos en Ecology of the Brain, donde sintetiza y desarrolla tanto sus propios trabajos previos sobre afectividad (Fuchs y Koch 2014, Fuchs 2013), como otras propuestas enactivas y corporizadas (Colombetti 2013, Sheets-Johnstone 1999, Damasio 1995). Fuchs propone que el sujeto cognitivo es un sujeto inherentemente afectivo en dos sentidos: primero, como parte inherente de su constitución como organismo vivo, $\mathrm{y}$, segundo, en sus interacciones con el mundo.

El primer sentido del afecto como constitutivo de la cognición está asociado a la causalidad vertical propia del ser vivo que regula sus procesos homeostáticos y que es fuente de la forma mínima de subjetividad y conciencia. De acuerdo con Fuchs, la organización misma del cuerpo como sistema vivo que se organiza y mantiene 
por sí solo implica tanto un sentido básico de autoafección, como la orientación afectiva hacia el mundo. En primer lugar, el sentido básico de autoafección integra el estado general del organismo con respecto a su autopreservación en cuanto organismo vivo (p. 60), e implica la sensación primordial de estar vivo, que toma la forma de fatiga o sensación de energía, bienestar o incomodidad, etc. Esta autoafección básica surge de los procesos de regulación vital que ocurren entre el organismo completo y el tallo cerebral, lo que contradice la visión dominante de la mente, en particular del sentido del yo como producto de la actividad del córtex. Fuchs respalda este punto reinterpretando múltiples investigaciones de Damasio (2010), Panksepp (1998) y Solms (2013) sin apelar a representaciones mentales o procesamientos de información localizados en el cerebro. Por ejemplo, en la hidranencefalia - condición en que los niños nacen sin cerebro, pero con tallo cerebral-, los niños:

no sólo están despiertos y conscientes, sino que también muestran claramente expresiones de sentimientos basales: gatean hacia lugares en el suelo donde la luz del sol está pegando, donde la niña se echará a disfrutar del sol y obviamente a beneficiarse del calor [...]; tienden a temer a extraños y mostrarse felices cerca de su madre/cuidador habitual $[\ldots]$; responden a diferentes sonidos instrumentales y voces humanas $[\ldots]$; se muestran de lo más alegres cuando los tocan y les hacen cosquillas, cuando sus piezas de música preferidas suenan y cuando se presentan ante sus ojos ciertos juguetes. (Damasio 2010, p. 81, citado en Fuchs 2018, pp. 113-114).

El sentido del yo que este caso extremo exhibe es precisamente la experiencia básica de primera persona de autoafección, es decir, ser y sentirse un cuerpo vivo en el mundo. No se trata de la activación de un sentido particular, tampoco de la percepción de un suceso o propiedad particular, y tampoco de una emoción porque no está dirigida a un objeto específico en el mundo que se evalúa.

Por otro lado, ser un cuerpo vivo implica estar orientado intencional y afectivamente hacia el mundo de acuerdo con el estado general del organismo; en otras palabras, parte de ser un cuerpo vivo es verse movido afectivamente a interactuar con el mundo. En su expresión más elemental, esta orientación toma la forma de impulsos básicos que empujan al organismo a perseguir lo que necesita, por ejemplo, por hambre, sed o deseo sexual — siguiendo a Spinoza, Fuchs llama a estos impulsos conatus (p. 71) —. La sensación de estar vivo y el conatus interactúan dinámicamente, y dependen de que exista la retroalimentación constante sobre el estado presente del organismo. Por 
ello, el afecto básico propio de los sujetos cognitivos es corporizado y no se podría explicar como un proceso esencialmente cerebral.

A pesar de la ubicuidad del afecto básico en la vida del sujeto corporizado, rara vez es objeto de su atención. La sensación de estar vivo y los impulsos básicos surgen a pesar de uno mismo, por ejemplo, la sensación de hambre. Es posible seguir o no estos impulsos, pero suelen permanecer como telón de fondo de otras funciones de la conciencia, en particular, aquellas dirigidas al mundo. De ahí proviene la sensación de pasividad que a veces está implícita en los afectos básicos y que surge a pesar de que son parte de la naturaleza propia del sujeto corporizado. Sin embargo, el afecto básico es la fuente y el centro de la vida mental del sujeto porque impulsa todas las funciones de la conciencia y, por ello, es el fundamento de la subjetividad; en otras palabras, constituye su yo más esencial. Por ser un afecto latente y corporizado, Fuchs en ocasiones se refiere al afecto básico como "el cuerpo profundo".

A diferencia del afecto básico, las emociones no sólo surgen de las dinámicas internas del organismo sino también de la relación del organismo con su ambiente, es decir, de sus procesos de causalidad horizontal, y son, además, mucho más prominentes en la atención y la conciencia que aquél. Éste es el segundo sentido en que los sujetos cognitivos son inherentemente afectivos. Para Fuchs, las emociones confieren significados vitales a objetos en el ambiente y la valencia del afecto básico se convierte en valores específicos de dichos objetos. Por ende, las emociones implican un componente distal, que es el objeto en el mundo al que están dirigidas, y un componente proximal, que se refiere a los procesos corporales verticales implícitos en la emoción (pp. 123-124). De esa manera, el afecto básico se convierte en un fenómeno dirigido al mundo y corporizado.

Esta capacidad de conferir valor a objetos en el mundo se desarrolla desde los primeros meses de vida en la interacción del bebé y su cuidador. A partir de la imitación de los gestos del adulto, el niño empieza a vivir experiencialmente precursores de la interacción emocional con otros, y, a partir de los nueve meses, comienza a asociar las expresiones emocionales de su cuidador con patrones en el ambiente (pp. 178-179). De manera decisiva, el bebé aprende a atender e interactuar con ciertos aspectos de su ambiente para regular su propio organismo, es decir, aprende a orientar sus propios afectos básicos. Esta interacción intersubjetiva es crucial para el desarrollo de las capacidades cognitivas del niño, y sienta las bases de su capacidad de interactuar con sentido con el mundo y con otros, y de vivir las 
emociones y los estados de ánimo complejos que caracterizan la vida humana. Por ello, para Fuchs, la interafectividad es fundamental en la cognición humana. Queda abierta, sin embargo, la pregunta acerca de cómo es exactamente la relación entre afectividad básica, por un lado, y patrones de interacción y orientación afectiva desarrollados en la interacción, por el otro. Si bien hay casos en los que los patrones interactivos permiten el mantenimiento del cuerpo profundo, es posible también que haya conflictos entre la afectividad del cuerpo profundo y los valores instaurados en la interacción. Esto apunta a una interrogante más general que sigue sin respuesta en el enactivismo, a saber, la pregunta por la relación entre cuerpo profundo e identidad sensoriomotora, sobre todo, en su aspecto sociocultural, que está siendo abordada, por ejemplo, en estudios sobre formación de hábitos y adicciones (Barandiaran 2008; Di Paolo et al. 2017; Ramírez-Vizcaya y Froese 2019).

Fuchs reconoce dos aspectos en la resonancia corporal propia de las emociones: por un lado, el aspecto afectivo, que se refiere a ser movido o tocado por el mundo a través de las sensaciones corporales, y por otro, el aspecto emotivo (e-movere), que se refiere a que las emociones nos "mueven a movernos" y por ello nos sentimos preparados para actuar. Ambos componentes surgen del ciclo interactivo entre afecto básico, percepción y movimiento. No hay que olvidar, sin embargo, que los ciclos propios de la emoción están dirigidos al mundo y no a una representación interna o a un estado cerebral del sujeto; por ello, para Fuchs, las emociones no se localizan en el interior del sujeto, sino que involucran tanto al sujeto como a las cualidades afectivas del ambiente.

La concepción de cognición en general y de emociones en particular que defiende Fuchs implica que no hay razón para diferenciarlas de otros procesos cognitivos tradicionalmente considerados de alto nivel y no afectivos (p. 110). Según Fuchs, la atención es una función de los procesos afectivamente cargados del organismo, y la acción sólo es posible si su conatus básico lo dispone afectivamente a anticipar cómo se sentirá corporalmente la acción que se llevará a cabo, y a ejecutarla, lo que implica la disposición fisiológica del cerebro y los músculos para la acción (p. 153); dicho de otro modo, la acción exige la disposición afectiva y corporizada del organismo.

\section{Emociones y otros procesos cognitivos}

La definición de emociones que propone Fuchs tiene una consecuencia aparentemente absurda que no se discute explícitamente en Ecol- 
ogy of the Brain, a saber, que todos los fenómenos cognitivos son emociones. Si asumimos, siguiendo a Fuchs, que la cognición consiste en interacciones del organismo vivo con su ambiente y además que su atención y sus acciones dentro de esta interacción surgen de su disposición a interactuar con aquello relevante para sus procesos afectivos, no queda claro cómo podríamos diferenciar un episodio emocional, como un abrazo de alegría, de otros episodios cognitivos, por ejemplo, escribir un argumento.

Fuchs sugiere un criterio de distinción en función del objeto al que se dirige el fenómeno mental: "las emociones emergen como formas específicas de la direccionalidad corporizada del sujeto hacia las cualidades afectivas y los valores de una situación dada", tales "cualidades afectivas del ambiente [son], por ejemplo, atractivo, cómodo, repulsivo, aterrador, extraño, etc." (p. 123). Sin embargo, si "la atención es una función de los propósitos y las motivaciones afectivamente cargadas del organismo" (p. 149) que se dirige a objetos específicos del ambiente a través de la actividad cortical, cualquier objeto del ambiente al que se dirija la atención estará cargado afectivamente para el organismo. En el ejemplo, ambos aspectos del ambiente a los que se dirige el sujeto son objetos cargados de valor para él: la persona abrazada es relevante para su vida emocional intersubjetiva y el argumento que escribe es relevante, probablemente, para sus intereses profesionales hacia los que sin duda dirige sus afectos. Así que, si seguimos la propuesta de Fuchs, caracterizar las emociones en función de la naturaleza afectiva de su objeto no es suficiente para diferenciar un fenómeno de otro: si ambas situaciones no movieran al sujeto afectivamente, es decir, en sus sensaciones y disposiciones corporales más vitales, y emotivamente, esto es, en su disposición a actuar de manera específica en el mundo, no se vería movido a actuar tal como lo hace.

Quiero sugerir que este aparente absurdo no apunta a un problema fundamental en la concepción de la cognición y las emociones de Fuchs; por el contrario, apunta a uno de los aspectos más originales que su libro aporta a la investigación enactiva. Para el enactivismo, la cognición surge del interés del organismo por mantenerse con vida, de manera que el ambiente se vuelve un mundo significativo para él porque lo amenaza o le permite continuar existiendo. Esta definición de cognición ha abierto la posibilidad, al enactivismo, de reconocer, desde sus primeras formulaciones, que la cognición tiene inherentemente un aspecto afectivo: el organismo no es indiferente a la forma en que el ambiente lo impacta y es movido por éste afectivamente (Varela y Depraz 2005, Varela et al. 1991). A pesar 
del reconocimiento temprano del papel fundamental de la afectividad, pocas investigaciones enactivas se han dedicado a profundizar en este punto, con excepción del trabajo de Colombetti (2013). Fuchs ofrece un esbozo lo suficientemente detallado para articular descripciones fisiológicas, cerebrales y fenomenológicas del papel esencial que el afecto tiene en la cognición, tanto en la constitución misma del sujeto cognitivo como en la forma en que el ambiente se convierte en un mundo de interacciones significativas para él. Si bien hay precursores que defienden explícitamente que la afectividad es esencial para la cognición en ciencias afectivas y neurología - entre ellos, Panksepp y Damasio - no existía, hasta ahora, una posición tan bien defendida empíricamente de esta idea desde un punto de vista corporizado y no cerebrocentrista.

El absurdo que señalé antes no es realmente tal porque todas las interacciones de un agente cognitivo y su ambiente son inherentemente afectivas y emocionales en la medida en que mueven sus afectos básicos y los mueven a actuar de maneras específicas en un mundo de valor para el agente. Pero dado el uso vernáculo del término "emoción", es sin duda contraproducente considerar que todas las formas de cognición son episodios emocionales, y es altamente probable que haya de hecho una diferencia no trivial entre los que consideramos episodios emocionales y no emocionales vernáculamente.

Sugiero una línea de investigación enactiva futura que parta de la hipótesis de que la diferencia entre lo que cotidianamente consideramos emociones y las demás formas de cognición no surge de una diferencia fisiológica o de las propiedades del objeto al que se dirige, sino de una diferencia esencialmente forjada por nuestras prácticas sociales. En su desarrollo, el niño aprende las circunstancias, los objetos y las expresiones apropiadas que en conjunto constituyen un episodio emocional. Nuestras prácticas compartidas hacen inteligible que, en ciertas circunstancias, un abrazo sea una manifestación de alegría, y en otras sea un saludo formal. Estos significados, siguiendo a Fuchs, están parcialmente incorporados en nuestros patrones fisiológicos e interactivos gracias a la mediación del córtex. La sedimentación neurofisiológica de patrones de interacción permite que nos acoplemos rápidamente a la interacción presente, y que la vivamos subjetivamente, bien como una emoción, o bien como una formalidad no emocional de interacción. Sin duda, Fuchs apunta en la dirección correcta al señalar que nuestra capacidad de aprender a vivir y reconocer emociones depende tanto del afecto básico inherente a nuestra constitución en cuanto sujetos cognitivos y corporizados, como de nuestro desarrollo intersubjetivo. Por mi parte planteo que el uso del 
lenguaje, en cuanto práctica social situada en contextos específicos y constantemente enactuada por una comunidad, es el elemento crucial que permitiría dar cuenta de qué constituye nuestras categorías de emoción, y de cómo se constituyen.

Actualmente existen varias líneas paralelas de investigación en emociones que enfatizan el papel de la comunidad y el lenguaje en la constitución de las emociones. Barrett (2006), por ejemplo, recopila resultados de los últimos treinta años de experimentos que intentan medir la correlación entre subgrupos de las siguientes categorías: movimientos faciales, señales vocales, cambios fisiológicos periféricos, acción voluntaria y experiencia subjetiva de emociones. A partir de allí muestra que, al contrario de lo que se esperaría si existieran emociones o afectos básicos, no se han encontrado grupos estables y confiables de respuesta; plantea que la razón es que las emociones básicas no existen, y argumenta, a partir del extenso trabajo experimental de su laboratorio, que las experiencias de emoción se configuran como unidades en virtud del aprendizaje de categorías conceptuales. Si bien la línea de investigación que sugiero a partir de la propuesta de Fuchs podría beneficiarse de sus resultados empíricos y del metaanálisis de datos en el que se basa la investigación de Barrett, no es necesario rechazar la existencia de emociones o afectos básicos, y mucho menos asumir que las emociones son categorías forjadas en el cerebro, para reconocer el papel constitutivo del lenguaje en nuestra vida emocional. En ese sentido y en una línea corporizada, situada y fenomenológica, trabajos como el de Slaby y colegas (von Scheve y Slaby 2019; Slaby et al. 2019) se han enfocado en la relación de mutua conformación entre colectivos sociales y repertorios emocionales que es evidente, por ejemplo, en situaciones de migración o en emociones colectivas (von Scheve e Ismer 2013), y muestran que entender "emoción" como categoría relacional permite analizar con más detalle estos fenómenos emocionales y profundamente sociales. La propuesta de Fuchs no sólo podría beneficiarse del trabajo interdisciplinario de Slaby, que pone en diálogo la filosofía y los estudios culturales del afecto, sino que también podría contribuir a éste sentando las bases neurofisiológicas de la definición de afecto como dinámica relacional en dominios sociales que propone Slaby.

De la mano de Fuchs, podemos aceptar la existencia de emociones básicas en cuanto patrones de procesos y reacciones organísmicas verticales y horizontales que nos disponen a sentir, por ejemplo, miedo, tristeza o alegría. Estos patrones se podrían entender como disposiciones corporizadas propias de nuestra constitución como humanos, 
mamíferos u organismos con sistema nervioso central. En investigaciones enactivas futuras, se debe profundizar en los detalles de la forma en que estos patrones se configuran, cuáles y cuántos son, y cómo se modifican intersubjetivamente en un dominio sociocultural durante el desarrollo. Esto daría vida a la propuesta enactiva de Hutto et al. (2018) de aceptar las emociones básicas concebidas como patrones corporizados, y permitiría, además, una reconciliación en ciencias afectivas que durante las últimas décadas se han anquilosado en la discusión bizantina en torno a si las emociones son innatas como emociones básicas o construidas como categorías sociales.

\section{BIBLIOGRAFÍA}

Barandiaran, X.E., 2008, Mental Life: A Naturalized Approach to the Autonomy of Cognitive Agents (tesis doctoral), Universidad del País Vasco (UPV-EHU), Donostia/San Sebastián. Disponible en: <https: //xabierbarandiaran.files.wordpress.com/2010/06/barandiaran_-_2008_phd_thesis.pdf $>$ [última consulta: 00/00/2019]

Barrett, L.F., 2006, Emotions as Natural Kinds?, Perspectives on Psychological Science, vol. 1, pp. 28-58.

Colombetti, G., 2013, The Feeling Body, MIT Press, Cambridge, Mass.

Damasio, A., 2010, Self Comes to Mind: Constructing the Conscious Brain, Pantheon Books, Nueva York.

—_, 1995, Descartes' Error: Emotion, Reason and the Human Brain, Picador, Londres.

De Kloet, E.R., M. Joëls, y F. Holsboer, 2005, "Stress and the Brain: From Adaptation to Disease", Nature Reviews Neurosciences, vol. 6, pp. 463475.

Di Paolo, E.A., T. Buhrmann, y X.E. Barandiaran, 2017, Sensorimotor Life: An Enactive Proposal, Oxford University Press, Oxford.

Fuchs, T., 2013, "Depression, Intercorporality and Interaffectivity", Journal of Consciousness Studies, vol. 20, pp. 219-238.

Fuchs, T. y S.C. Koch, 2014, "Embodied Affectivity: On Moving and Being Moved", Frontiers in Psychology. Psychology for Clinical Settings, vol. 5, no. 508. (doi:10.3389/fpsyg.2014.00508)

Hutto, D.D., I. Robertson, y M.D. Kirchhoff, 2018, “A New, Better BET: Rescuing and Revising Basic Emotion Theory", Frontiers in Psychology, vol. 9, no. 1217. (doi:10.3389/fpsyg.2018.01217)

Kelso, J.A.S., B. Tuller, E. Vatikiotis-Bateson, y C.A. Fowler, 1984, "Functionally Specific Articulatory Cooperation Following Jaw Perturbations During Speech: Evidence for Coordinative Structures", Journal of Experimental Psychology: Human Perception and Performance, vol. 10, no. 6, pp. 812-832. (doi:10.1037/0096-1523.10.6.812) 
Panksepp, J., 1998, Affective Neuroscience: The Foundations of Human and Animal Emotions, Oxford University Press, Oxford.

Ramírez-Vizcaya, S., y T. Froese, 2019, "The Enactive Approach to Habits: New Concepts for the Cognitive Science of Bad Habits and Addiction", Frontiers in Psychology, vol. 10, no. 301. (doi:10.3389/fpsyg.2019.00301)

Sheets-Johnstone, M., 1999, "Emotion and Movement: A Beginning Empirical-Phenomenological Analysis of Their Relationship", Journal of Consciousness Studies, vol. 6, nos. 11-12, pp. 259-277.

Slaby, J.,R. Mühlhoff, y P. Wüschner, 2019, "Affective Arrangements", Emotion Review, vol. 11, no. 1, pp. 3-12.

Solms, M., 2013, "The Conscious Id", Neuropsychoanalysis: An Interdisciplinary Journal for Psychoanalysis and the Neurosciences, vol. 15, pp. 5-19.

Varela, F.J., y N. Depraz, 2005, "At the Source of Time: Valence and the Constitutional Dynamics of Affect", Journal of Consciousness Studies, vol. 12, nos. 8-10, pp. 61-81.

Varela, F.J., E. Thompson, y E. Rosch, 1991, The Embodied Mind: Cognitive Science and Human Experience, MIT Press, Cambridge, Mass.

Von Scheve, C. y J. Slaby, 2019, "Emotion, Emotion Concept", en Slaby y von Scheve (comps.), Affective Societies: Key Concepts, Routledge, Abingdon/ Nueva York.

Von Scheve, C. y S. Ismer, 2013, "Towards a Theory of Collective Emotions", Emotion Review, vol. 5, no. 4, pp. 406-413.

Recibido el 22 de agosto de 2019; revisado el 13 de febrero de 2020; aceptado el 20 de febrero de 2020. 